\title{
Patrones de distribución de macroalgas y macroinvertebrados intermareales de la isla Robinson Crusoe, archipiélago de Juan Fernández, Chile
}

\author{
María Eliana Ramírez ${ }^{1}$ y Cecilia Osorio ${ }^{2}$ \\ ${ }^{1}$ Laboratorio de Algas Marinas, Museo Nacional de Historia Natural \\ Casilla 787, Santiago, Chile \\ E-mail: mramirez@mnhn.cl \\ ${ }^{2}$ Departamento de Ciencias Ecológicas, Facultad de Ciencias. Universidad de Chile \\ Casilla 653, Santiago, Chile \\ E-mail: cosorio@abello.dic.uchile.cl
}

Recibido 20 diciembre 1998; versión corregida 20 abril 1999; aceptado 10 junio 1999

\begin{abstract}
RESUMEN. El presente trabajo describe cualitativa y cuantitativamente los patrones de distribución de los organismos sésiles del intermareal rocoso de la isla Robinson Crusoe. Los datos cualitativos corresponden a observaciones realizadas en el terreno durante tres exploraciones a la isla. Los datos cuantitativos fueron obtenidos en base a la medición de la cobertura de organismos a lo largo de un transecto vertical de 11,6 m, extendido sobre toda la zona intermareal en la localidad de playa El Palillo. Los resultados obtenidos permiten señalar el siguiente patrón de distribución para estas comunidades de sustratos duros. En el intermareal alto (2 a $3 \mathrm{~m}$ ), la única especie presente es Nodilittorina fernandezensis, con coberturas de 14 a 17\%. En el intermareal medio (1 a $2 \mathrm{~m}$ ) las especies dominantes son Chaetomorpha firma (62\%), Ulva rigida (36\%) y Gelidium pseudointricatum (16\%). Entre los invertebrados presentes en este nivel destacan Jehlius cirratus (10\%) y Heliaster canopus, presente sólo a $1 \mathrm{~m}$ de altura intermareal con un $21 \%$ de la cobertura. En el intermareal bajo dominan nuevamente las algas. Entre 0,51 y $1 \mathrm{~m}$ son dominantes en cobertura Corallina officinalis (27\%), Chondracanthus intermedius (20\%) y G. pseudointricatum (12 a $13 \%)$. En los niveles mareales inferiores a $0,51 \mathrm{~m}$ las especies dominantes son Ulva rigida (23\%) y Sphacelaria cirrosa (20\%). Los patrones de distribución ecológica de estas comunidades son diferentes de aquéllos descritos para comunidades cercanas del continente. Factores físicos como la pronunciada pendiente de los roqueríos y biogeográficos, propios de este archipiélago serían determinantes en la composición de especies y en la estructura de estas comunidades.
\end{abstract}

Palabras claves: distribución, macroalgas, macroinvertebrados, intermareal rocoso, archipiélago de Juan Fernández, Chile.

\section{Distributional patterns of the intertidal macroalgae and macroinvertebrates from Robinson Crusoe island, Juan Fernandez archipelago, Chile}

\begin{abstract}
The present work describe in qualitative and quantitative terms the distributional patterns of the marine organisms from a rocky shores in the "El Palillo" beach at Robinson Crusoe Islands Archipelago Juan Fernandez, in base to a new exploration in the field and also in base to a coberture percentage of the organisms over a transect of 11.6 $\mathrm{m}$. The following distributional patterns was observed. In the upper intertidal zone the small mollusk Nodilittorina fernandezensis is present with a coberture of 14 and 17\%. In the middle intertidal zone (1 to $2 \mathrm{~m}$ ) the dominants species are Chaaetomorpha firma (62\%), Ulva rigida (36\%) and Gelidium pseudointricatum (16\%). Between the invertebrates at this tidal level we found Jehlius cirratus (10\%) and Heliaster canopus ( $21 \%$ ) only at $1 \mathrm{~m}$. In the low intertidal the algae Corallina officinalis (27\%), Chondracanthus intermedius (20\%) and G. pseudointricatum (12 to $13 \%)$ are the dominants species. Below this level the species Ulva rigida $(23 \%)$ and Sphacelaria cirrosa $(20 \%)$ are the dominants. These patterns are different to those described for others near communities of the continent. Physical and biogeographicals factors can be determinants in the species composition and the structure of these communities.
\end{abstract}

Key words: distribution, macroalgae, macroinvertebrates, rocky intertidal habitat, Juan Fernandez archipelago, Chile. 


\section{INTRODUCCIÓN}

El archipiélago de Juan Fernández (3340'S; $79^{\circ} 00^{\prime} \mathrm{W}$ ), situado aproximadamente a $700 \mathrm{~km}$ de la costa de Chile continental frente al Puerto de San Antonio, Chile, constituye uno de los territorios insulares de mayor interés científico por la importancia biológica y biogeográfica de su biota. Las islas de Juan Fernández son consideradas como una de las áreas protegidas del Estado chileno, declarada como Parque Nacional desde 1935 y Reserva Mundial de la Biosfera por UNESCO desde 1977.

Los esfuerzos de estudio en este archipiélago se han concentrado preferentemente en el ecosistema terrestre y en menor grado en las comunidades y ambiente marino, a pesar que elementos de esta biota son los que en la actualidad sustentan la economía y sobrevivencia de la población insular.

Las dificultades de acceso a estas islas desde el continente, así como la escasa accesibilidad a sus accidentadas costas, han sido limitantes en el conocimiento de los organismos marinos y de las comunidades de las cuales ellos forman parte. Los antecedentes reunidos a la fecha indican que la mayor parte del conocimiento de la biota marina del archipiélago corresponde a estudios puntuales sobre determinados organismos de importancia económica para la isla, como la langosta de Juan Fernández Jasus frontalis Milne Edwards (Arana, 1987) y a trabajos taxonómicos que dan cuenta de las principales especies de la flora y fauna marina, producto de recolecciones realizadas por expediciones naturalísticas durante el siglo pasado y en el presente siglo. De éstas se destacan la expedición británica del H.M.S. Challenger (1872-1876) y la Expedición sueca del Pacífico (Swedish Pacific Expedition) (1916-1917). El desarrollo histórico de este conocimiento se encuentra resumido en los trabajos de Santelices (1987), y Rozbaczylo y Castilla (1987). Es importante destacar entre estos trabajos la obra de Levring (1941) y posteriormente las contribuciones de Earle (1969), Müller y Ramírez (1994) y Ramírez (1995) en algas; Odhner (1922) en moluscos; Desqueyroux (1972) en poríferos; Codoceo (1976) en equinodermos, y Báez y Ruiz (1985) en crustáceos.

Las comunidades marinas de las costas rocosas del archipiélago han sido escasamente estudiadas. A excepción del trabajo de Skottsberg (1943) quien describe de manera cualitativa las principales asociaciones de algas del intermareal de la costa sur de la isla Robinson Crusoe y de Etcheverry (1960) quien da a conocer también cualitativamente la distribución de algas en el sublitoral poco profundo de bahías protegidas del archipiélago, no existe otra información sobre el tema.

El presente estudio describe en forma cualitativa y cuantitativa los patrones de distribución y principales asociaciones de organismos marinos del intermareal rocoso de la isla Robinson Crusoe con el propósito de conocer la estructura de estas comunidades.

\section{MATERIALES Y MÉTODOS}

Se realizaron 10 muestreos cualitativos destinados a la recolección de todos los taxa presentes y a describir las principales asociaciones de la flora y fauna marina del intermareal rocoso y submareal somero, en diferentes localidades de la isla de Robinson Crusoe entre enero y marzo de 1996.

Se realizó un muestreo cuantitativo sobre la base de la cobertura, en la localidad de playa El Palillo el 22 de enero de 1996, en el sector norte de Bahía Cumberland, que es uno de los pocos roqueríos accesibles para realizar este tipo de trabajo (Fig. 1). La medición de la cobertura de organismos sésiles (macroinvertebrados y macroalgas) se realizó mediante el método de intersección de puntos sobre cuadrantes reticulados de $400 \mathrm{~cm}^{2}$, dispuestos sobre un transecto vertical de $11,6 \mathrm{~m}$, orientado en forma perpendicular a la línea de costa, extendido desde el límite inferior de la línea de más baja marea. No se pudo realizar una réplica de este transecto por las dificultades topográficas de la costa.

Se determinó la densidad de individuos (indiv./ $\mathrm{m}^{2}$ ), para describir los patrones de abundancia de algunos invertebrados presentes mediante cuadrantes lanzados al azar, de $1 \mathrm{~m}^{2}$ para holoturias y de $20 \times 20 \mathrm{~cm}$ para gastrópodos. En ambos casos estos muestreos se replicaron 10 veces. Todo el material recolectado se depositó en las colecciones de Algas Marinas del Herbario Nacional (SGO) y en las colecciones hidrobiológicas del Museo Nacional de Historia Natural de Santiago.

\section{RESULTADOS}

La mayor parte de la isla Robinson Crusoe es homogénea en paisajes. En general, tiene una costa muy expuesta, con roqueríos de pendiente pronunciada y una zona intermareal estrecha, la que se hace 


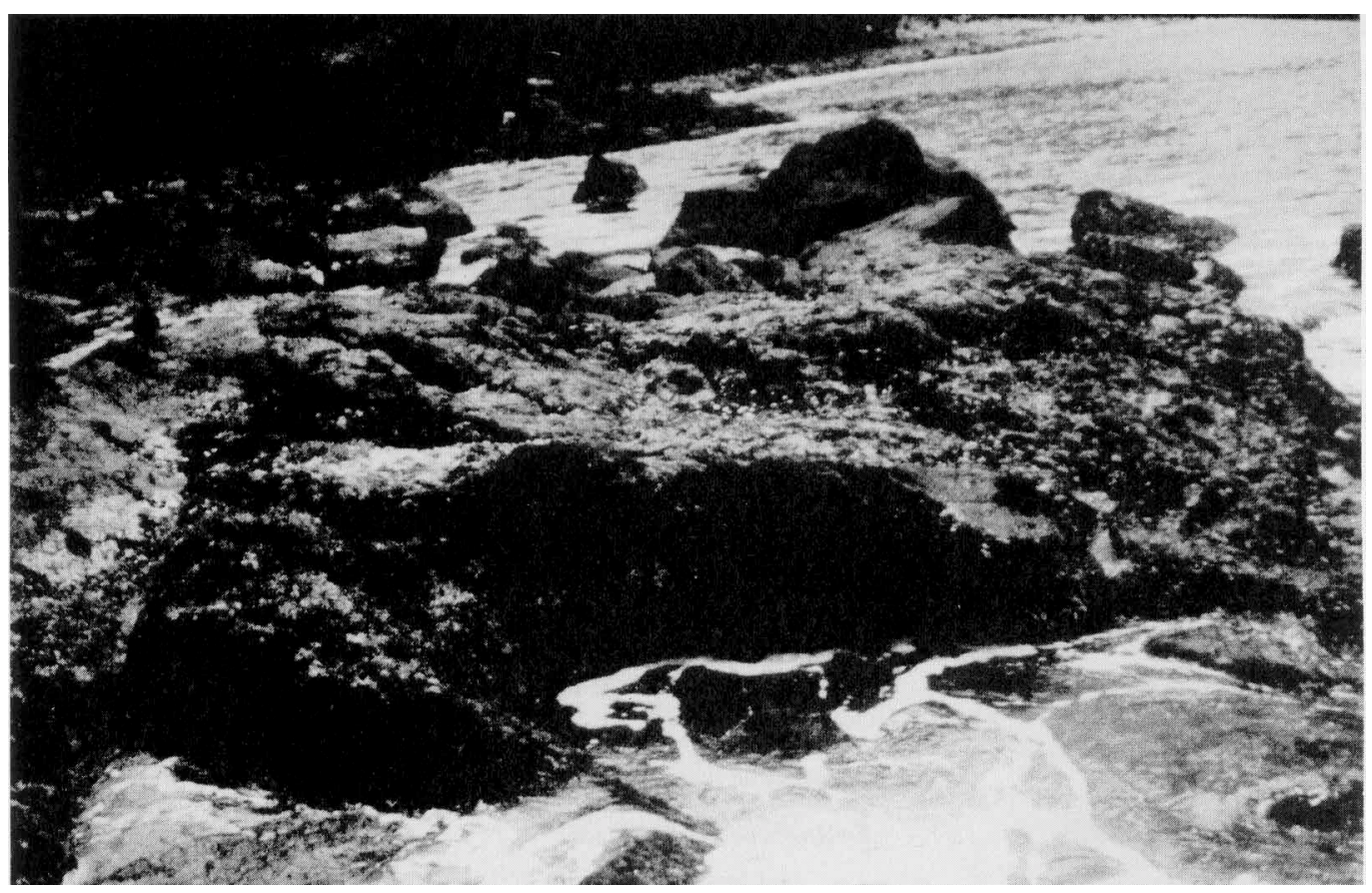

Figura 1. Intermareal Rocoso en playa El Palillo, isla Robinson Crusoe.

Figure 1. Intertidal rocky shore in El Palillo beach, Robinson Crusoe island.

evidente sólo en algunas playas del sector norte (Puerto Inglés, El Palillo, El Pangal, Puerto Francés). El sustrato rocoso comprende rocas desmembradas de distintos tamaños con algunos bolones, las costas menos expuestas son reducidas y están representadas por bahías protegidas, como bahía del Padre y Bahía Cumberland. Esta última presenta ambientes protegidos y semi-expuestos, con playas de cantos rodados de poca pendiente y escasamente colonizadas por organismos, y roqueríos de pendiente más pronunciada con rocas más resistentes pero siempre desmembradas, donde crece y se desarrolla una flora y fauna bentónica. El sustrato rocoso es de roca volcánica donde predominan los flujos de lava sobre otros depósitos (González-Ferrán, 1987).

Un esquema de la distribución de los principales organismos marinos que habitan el intermareal rocoso en playa El Palillo se indica en la Figura 2. Al menos 12 especies de invertebrados y 9 de macroalgas se identificaron fácilmente. Un análisis taxonómico más fino permitió la identificación de 28 invertebrados y 47 macroalgas bentónicas (Tablas 1 y 2).

De los macroinvertebrados recolectados en el intermareal de playa El Palillo (Tabla 2), la mayoría tiene registros anteriores en el área y 13 especies son endémicas. El poliqueto Paleanotus versicolor, endémico de Chile continental, conocido previamente entre las localidades de Antofagasta y Coquimbo se registra por primera vez en el archipiélago. Con respecto a moluscos sólo 10 de las 43 especies citadas para el archipiélago fueron recolectadas en los muestreos. Los gastrópodos Nodilittorina fernandezensis y Aplysia parvula juanina se encontraron en período reproductivo. Algunos ejemplares pequeños (menores de $1 \mathrm{~cm}$ ) de Plaxiphora fernandenzi fueron recolectados entre las algas, junto a isópodos y anfípodos. De los 13 equinodermos citados para el archipiélago (Codoceo, 1976), sólo tres aparecieron en los muestreos, todos endémicos para el archipiélago.

Las algas recolectadas en El Palillo (Tabla 1), corresponden a especies citadas anteriormente para la isla (Levring, 1941), sólo Halymenia floridana se registra por primera vez para la localidad y constituye el primer registro para las costas del hemisferio sur, siendo conocida previamente en las costas tropicales del Atlántico oeste. Es importante señalar el alto número de especies endémicas de este archipiélago, muchas de las cuales son de tamaño 
Tabla 1. Macroalgas del intermareal rocoso de playa El Palillo, isla Robinson Crusoe. Enero-Marzo 1996. Table 1. Macroalgae in the intertidal rocky shore in El Palillo beach, Robinson Crusoe island. January-March 1996.

\begin{tabular}{|c|c|c|}
\hline TAXA & DISTRIBUCIÓN GEOGRAFICA & OBSERVACIONES \\
\hline \multicolumn{3}{|l|}{ Chlorophyta } \\
\hline Ulva rigida $\mathrm{C}$. Ag. & Amplia distribución & Intermareal \\
\hline Enteromorpha compressa (L.) Grev. & Amplia distribución & Intermareal, en pozas \\
\hline Chaetomorpha linum (O.F. Müll.) Kütz. & Amplia distribución & Intermareal \\
\hline Chaetomorpha firma Levr. & $\begin{array}{l}\text { Chile continental, Isla de Pascua, } \\
\text { Desventuradas y Juan Fernández }\end{array}$ & Intermareal \\
\hline Cladophora perpusilla Sköttsb. y Levr. & Endémica Juan Fernández e Isla de Pascua & Intermareal \\
\hline Rhizoclonium tortuosum (Dillw.) Kütz. & Amplia distribución & Intermareal y submareal \\
\hline Mycrodictyon japonicum Setch. & $\begin{array}{l}\text { Mares temperados cálidos de Asia, } \\
\text { Océano Indico, isla de Pascua }\end{array}$ & $\begin{array}{l}\text { Epífita en otras algas del } \\
\text { intermareal y submareal somero }\end{array}$ \\
\hline Codium cerebriforme Setch. & Endémica de Juan Fernández & Submareal hasta 3-4 m. \\
\hline Codium fernandezianum Setch. & Endémica de Juan Fernández & Submareal, colectada varada en la playa \\
\hline Bryopsis pennata Lamour. & De amplia distribución en mares cálidos & Intermareal y submareal somero \\
\hline \multicolumn{3}{|l|}{ Phaeophyta } \\
\hline Hincksia granulosa (Smith) Silva & Amplia distribución en mares temperados & $\begin{array}{l}\text { Epífita en grandes algas, } \\
\text { intermareal y submareal somero }\end{array}$ \\
\hline Hincksia mitchelliae (Harv.) Silva & Amplia distribución en mares temperados & $\begin{array}{l}\text { Epífita en otras algas. } \\
\text { Intermareal y submareal somero }\end{array}$ \\
\hline Myrionema strangulans Grev. & Amplia distribución & Epífita en Ulva spp. \\
\hline Scytothamnus australis (J. Ag.) Hook y & Subantártica & Intermareal medio-alto \\
\hline Splachnidium rugosum (L.) Grev. & Subantártica & Intermareal \\
\hline Sphacelaria cirrosa (Roth) C. Ag. & Amplia distribución mares temperados cálidos & Intermareal y submareal somero \\
\hline Dictyota phlyctaenodes Mont. & Endémica Juan Fernández y Desventuradas & Intermareal bajo a submareal somero \\
\hline Glossophora künthii (C. Ag.) J. Ag. & Subantártica & Intermareal bajo a submareal somero \\
\hline Padina fernandeziana Skötts y Levr. & Endémica de Juan Fernández & Intermareal bajo a submareal somero \\
\hline Distromium skottsbergii Levr. & Subantártica & Intermareal en pozas de marea \\
\hline Colpomenia durvillaei (Bory) Ramírez & $\begin{array}{l}\text { Endémica a la costa de } \\
\text { Chile centro-sur y Juan Fernández }\end{array}$ & Intermareal \\
\hline $\begin{array}{l}\text { Colpomenia sinuosa (Mertens ex Roth) } \\
\text { Dérb. et Sol. }\end{array}$ & Amplia distribución & Intermareal a submareal somero \\
\hline
\end{tabular}




\begin{tabular}{|c|c|c|}
\hline TAXA & DISTRIBUCIÓN GEOGRAFICA & OBSERVACIONES \\
\hline \multicolumn{3}{|l|}{ Rhodophyta } \\
\hline Acrochaetium fernandezianum Levr. & Endémica de Juan Fernández & Epífita en Codium fernandezianum \\
\hline Liagora brachyclada Mont. & Endémica de Juan Fernández & Intermareal bajo a submareal somero \\
\hline Gelidium lingulatum Kütz. & Endémica de Chile continental, Juan Fernández & Intermareal bajo \\
\hline Gelidium pseudointrincatum Skottsb. y L & Levr. Endémica de Juan Fernández & Intermareal \\
\hline Corallina officinalis var. Chilensis (Dec.) & Amplia distribución & Intermareal \\
\hline Halymenia floridana J.Ag. & Atlántico Tropical Oeste & Intermareal y submareal somero \\
\hline Chondracanthus intermedius (Suhr) Fred & Amplia distribución mares cálidos & Intermareal bajo \\
\hline Ahnfeltiopsis furcellatus (C. Ag.) Silva & Subantártica & Intermareal \\
\hline Chondriella pusilla Levr. & Endémica de Juan Fernández & Intermareal \\
\hline Gloioderma subdichotoma Levr. & Endémica de Juan Fernández & Submareal somero \\
\hline Antithamnion minutissimun Levr. & Endémica de Juan Fernández & $\begin{array}{l}\text { Epífita en algas. } \\
\text { Intermareal y submareal somero }\end{array}$ \\
\hline Ceramiun rubrum (Huds.) C. Ag. & Amplia distribución & Intermareal y submareal somero \\
\hline Centroceras clavulatum (C. Ag.) Mont. & Amplia distribución & Intermareal \\
\hline Griffithsia sp. & & Intermareal y submareal somero \\
\hline Branchioglossum parvulum Levr. & $\begin{array}{l}\text { Endémica de Juan Fernández y } \\
\text { Desventuradas }\end{array}$ & $\begin{array}{l}\text { Epífita en otras algas. } \\
\text { Intermareal y submareal somero }\end{array}$ \\
\hline Hymenena decumbens Levr. & Subantártica & $\begin{array}{c}\text { Epífita en otras algas. } \\
\text { Intermareal y submareal somero }\end{array}$ \\
\hline Dasya sp. & & Epífita en otras algas. Intermareal \\
\hline Pterosiphonia dendroidea (Mont.) Falk. & Amplia distribución mares temperados & Epífita en algas. Intermareal y submareal \\
\hline Pterosiphonia disticha Levr. & $\begin{array}{c}\text { Chile continental, Juan Fernández, } \\
\text { Desventuradas }\end{array}$ & $\begin{array}{l}\text { Epífita algas intermareales y } \\
\text { del submareal somero }\end{array}$ \\
\hline Pterosiphonia pusilla Levr. & Endémica de Juan Fernández y Desventuradas & Epífita. Intermareal y submareal somero \\
\hline $\begin{array}{l}\text { Dipterosiphonia parva (Dick.) } \\
\text { Skottsb. y Levr. }\end{array}$ & $\begin{array}{c}\text { Endémica Juan Fernández } \\
\text { y Desventuradas }\end{array}$ & $\begin{array}{c}\text { Epífita. Intermareal } \\
\text { y submareal somero. Abundante }\end{array}$ \\
\hline Fernandosiphonia unilateralis Levr. & Endémica, Juan Fernández y Desventuradas & $\begin{array}{l}\text { Epífita de manera abundante } \\
\text { en diferentes algas tanto en el } \\
\text { Intermareal como submareal somero } \\
\text { Intermareal en pozas de marea }\end{array}$ \\
\hline Stictosiphonia Hookeríi Hook. & Subantártica & Intermareal \\
\hline Levringiella microscopicus (Levr.) Kylin & Endémica de Juan Fernández & Epífita. Intermareal y submareal somero \\
\hline
\end{tabular}


Tabla 2. Macroinvertebrados del intermareal rocoso en playa El Palillo, isla Robinson Crusoe. Enero- Marzo 1996.

Table 2. Macroinvertebrates in the intertidal rocky shore in EI Palillo beach, Robinson Crusoe island. JanuaryMarch 1996.

\begin{tabular}{|c|c|c|}
\hline TAXA & DISTRIBUCIÓN GEOGRAFICA & OBSERVACIONES \\
\hline \multicolumn{3}{|l|}{ ANTHOZOA } \\
\hline Phymactis clematis (Drayton, 1846) & $\begin{array}{l}\text { California, Golfo de Panamá costas Ecuador, } \\
\text { Perú, Chile y Archipiélago de Juan Fernández }\end{array}$ & Intermareal \\
\hline \multicolumn{3}{|l|}{ ANNELIDA POLYCHAETA } \\
\hline Perinereis camiguinoides (Augener, 1922) & Isla Robinson Crusoe & Intermareal hasta $35 \mathrm{~m}$ \\
\hline Perinereis. Pseudocamiguina (Augener, 1922) & $\begin{array}{l}\text { Coquimbo, } \\
\text { Archipiélago de Juan Fernández }\end{array}$ & Intermareal \\
\hline Lepidonotus versicolor Ehlers, 1901 & Archipiélago de Juan Fernández & Intermareal hasta $45 \mathrm{~m}$ \\
\hline Typosyllis sp. & & Intermareal \\
\hline Lysidice trimera Ehlers, 1901 & Archipiélago de Juan Fernández & Intermareal \\
\hline Marphysa aenea (Blanchard, 1849) & $\begin{array}{l}\text { Iquique hasta Archipiélago Chonos y } \\
\text { Archipiélago de Juan Fernández }\end{array}$ & Intermareal hasta $45 \mathrm{~m}$ \\
\hline Arabella iricolor caerulea (Schmarda, 1861) & $\begin{array}{l}\text { Coquimbo a Golfo de Ancud, } \\
\text { Archipiélago de Juan Fernández }\end{array}$ & Intermareal hasta $35 \mathrm{~m}$ \\
\hline $\begin{array}{l}\text { Paleanotus chrysolepis Schmarda, } 1861 \\
\text { MOLLUSCA }\end{array}$ & Mejillones, Antofagasta & 0 a $0,5 \mathrm{~m}$ \\
\hline Bivalvia & & \\
\hline Arca (A) fernandezensis Hertlein y Strong, 1943 & $\begin{array}{l}\text { Archipiélago } \\
\text { de Juan Fernández }\end{array}$ & Valvas \\
\hline Chama pellucida Sowerby, 1834 & $\begin{array}{l}\text { Archipiélago de Juan Fernández, } \\
\text { California a Valparaíso }\end{array}$ & Intermareal bajo \\
\hline Lasaea macrodon Stempell, 1899 & Archipiélago de Juan Fernández & Entre algas \\
\hline Modiolus aurum Osorio, 1979 & Archipiélago de Juan Fernández & Valvas \\
\hline Gastropoda & & \\
\hline Acmaea juanina Odhner, 1921 & Archipiélago de Juan Fernández & Intermareal \\
\hline Nodilittorina fernandezensis Rosewater, 1970 & $\begin{array}{c}\text { Archipiélago } \\
\text { de Juan Fernández }\end{array}$ & Supramareal \\
\hline Diloma crusoeana (Pilsbry, 1889) & $\begin{array}{c}\text { Archipiélago de Juan Fernández, } \\
\text { Pascamayo (Perú) a Coquimbo (Chile). }\end{array}$ & Intermareal \\
\hline
\end{tabular}




\begin{tabular}{|c|c|c|}
\hline TAXA & DISTRIBUCIÓN GEOGRAFICA & OBSERVACIONES \\
\hline \multicolumn{3}{|l|}{ Concholepas concholepas fernandezianus } \\
\hline Bruguière, 1789 & Archipiélago de Juan Fernández & Valvas \\
\hline Aplysia parvula juanina (Bergh, 1898) & Archipiélago de Juan Fernández & Intermareal \\
\hline Serpulorbis sp. & & Intermareal \\
\hline \multicolumn{3}{|l|}{ Polyplacophora } \\
\hline Plaxiphora fernandezi Thiele, 1909 & Archipiélago de Juan Fernández & Intermareal \\
\hline \multicolumn{3}{|l|}{ Cephalopoda } \\
\hline Octopus sp. & & Intermareal inferior \\
\hline \multicolumn{3}{|l|}{ CRUSTACEA } \\
\hline Jehlius cirratus Darwin & Chile continental, Isla Robinson Crusoe & Intermareal superior \\
\hline Leptograpsus variegatus (Fabricius, 1793) & Amplia distribución geográfica & Intermareal \\
\hline \multicolumn{3}{|l|}{ Anphipoda } \\
\hline \multicolumn{3}{|l|}{ Isopoda } \\
\hline \multicolumn{3}{|l|}{ ECHINODERMATA } \\
\hline Heliaster canopus Perrier, 1875 & I. Desventuradas y Archipiélago de Juan Fernández & Intermareal \\
\hline Patiriella calcarata (Perrier, 1869) & Archipiélago de Juan Fernández & Intermareal \\
\hline Mertensiothuria platei (Ludwig) & Archipiélago de Juan Fernández & Intermareal \\
\hline
\end{tabular}




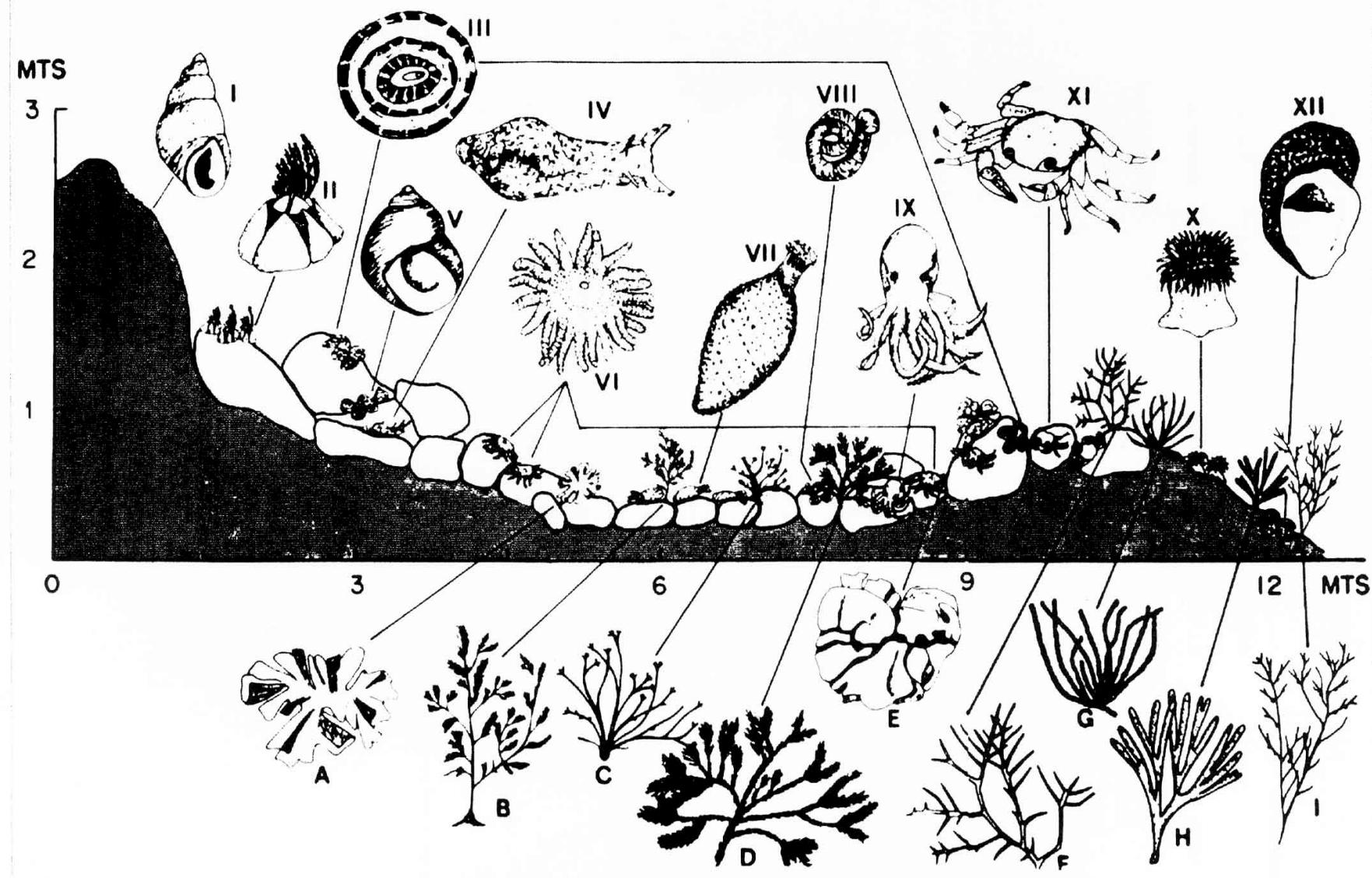

Figura 2. Esquema de distribución de los principales organismos marinos del intermareal rocoso en playa El Palillo, isla Robinson Crusoe.

Figure 2. Distribution of the main marine organisms on the intertidal rocky shore in El Palillo beach, Robinson Crusoe island.

I Nodilittorina fernandezensis. II Jelhius cirratus. III Acmaea juanina. IV Aplysia parvula juanina. V Diloma crusoena. VI Heliaster canopus. VII Mertensiothuria platei. VIII Vermetidae. IX Octopus sp. X Phymactis clematis. XI Leptograpsus variegatus. XII Chama pellucida.

A Ulva rigida. B Scytothamnus australis. C Gelidium pseudointricatum. D Corallina officinalis var. chilensis. E Colpomenia sinuosa.F Chondracanthus intermedius. G Chaetomorpha firma. H Dictyota phlyctaeonodes. I Sphacelaria cirrosa. 
pequeño y crecen como epífitas en otras algas. El alto número de taxa identificados constituye una muestra representativa de las algas del archipiélago (alrededor de un 50\%), si se considera que el número total y real de especies presentes es del orden de las 100 especies. Se considera 100 como número real a pesar que en la actualidad se sabe que el registro total de especies se eleva a 123 (Ramírez, 1999, en prensa). Esto debido a que muchos de estos registros no han sido confirmados con posteridad, de manera que alrededor de un $20 \%$ de esta información debería ser considerada insustancial.

$\mathrm{El}$ análisis de cobertura medida en terreno (Fig. 3 ), muestra que el intermareal alto entre 2 y $3 \mathrm{~m}$, presenta entre un 80 a $90 \%$ de espacio primario libre, encontrándose el resto del sustrato ocupado sólo por invertebrados. Entre 1 a $2 \mathrm{~m}$ de altura intermareal disminuye el porcentaje de roca desnuda, repartiéndose el espacio macroinvertebrados, algas y un sinnúmero de invertebrados de menor tamaño que cohabitan con las algas. Hacia los niveles más bajos de la zona intermareal ( 1 a $0,5 \mathrm{~m}$ ), el sustrato es ocupado preferentemente por algas con porcentajes de cobertura entre un 70 a $80 \%$.

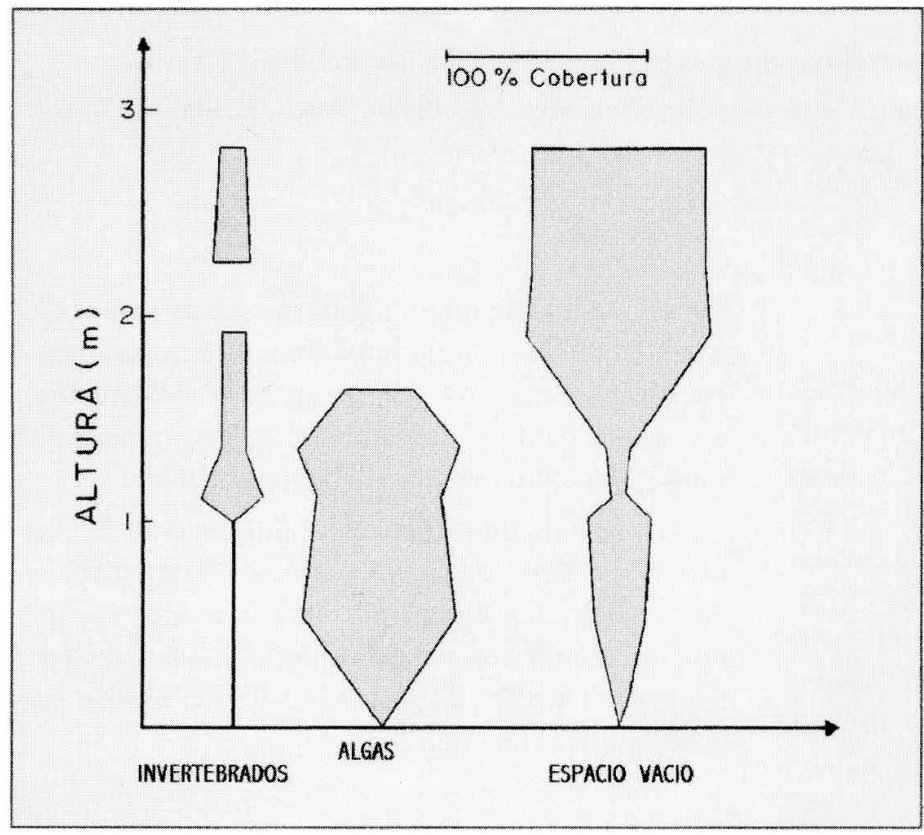

Figura 3. Valores de cobertura de macroalgas y macroinvertebrados en el intermareal rocoso de playa EI Palillo, isla Robinson Crusoe.

Figure 3. Cover values of macroalgae and macroinvertebrates in the intertidal rocky Shore in El Palillo beach, Robinson Crusoe island.
La distribución de organismos según su cobertura (Fig. 4), permite describir el siguiente patrón de zonación. En el intermareal alto ( 2 a 3 m) la única especie presente es Nodilittorina fernandezensis que es endémica de la isla, con coberturas de 14 a $17 \%$. En el intermareal medio ( 1 a $2 \mathrm{~m}$ ) el sustrato está colonizado por varias especies de macroalgas con valores variables de cobertura siendo dominantes las especies Chaetomorpha firma (62\%), Ulva rigida (36\%) y Gelidium pseudointricatun (16\%). Entre los invertebrados destacan Jehlius cirratus y Heliaster canopus. El cirripedio J. cirratus muestra un porcentaje de cobertura relativamente bajo (10\%) que se mantiene constante a lo largo de su distribución en esta zona, para luego desaparecer casi completamente a $1 \mathrm{~m}$ de altura intermareal. $H$. canopus aparece sólo a $1,1 \mathrm{~m}$ con un $21 \%$ de cobertura. En el límite superior del intermareal bajo $(0,5$ a $1 \mathrm{~m})$, las especies dominantes en cobertura son las algas rojas Corallina officinalis var. chilensis (27\%), Chondracanthus intermedius (20\%) y Gelidium pseudointricatum (12 a 13\%). Hacia los niveles intermareales inferiores a $0,51 \mathrm{~m}$ de altura intermareal, las especies dominantes son Ulva rigida (23\%) y Sphacelaria cirrosa (20\%). En esta franja media e inferior dominada por diferentes algas de tamaño pequeño a medio, algunas especies como Gelidium y Chondracanthus crecen sobre las rocas a manera de césped formando colchones afelpados que retienen bastante agua. Entre estas algas habita un sinnúmero de pequeños invertebrados como poliquetos, nemátodos, y algunos isópodos y anfípodos que las utilizan de hábitat, alimento, refugio contra los depredadores o de protección contra la desecación en las horas de exposición al aire.

Con respecto a las densidades específicas medidas en el terreno se puede señalar que Nodilittorina fernandezensis registró densidades entre 65 a 430 indiv. $/ \mathrm{m}^{2}$ y Metensiothuria platei que es abundante en las pozas litorales, presentó densidades de 20 a 25 indiv. $/ \mathrm{m}^{2}$. 


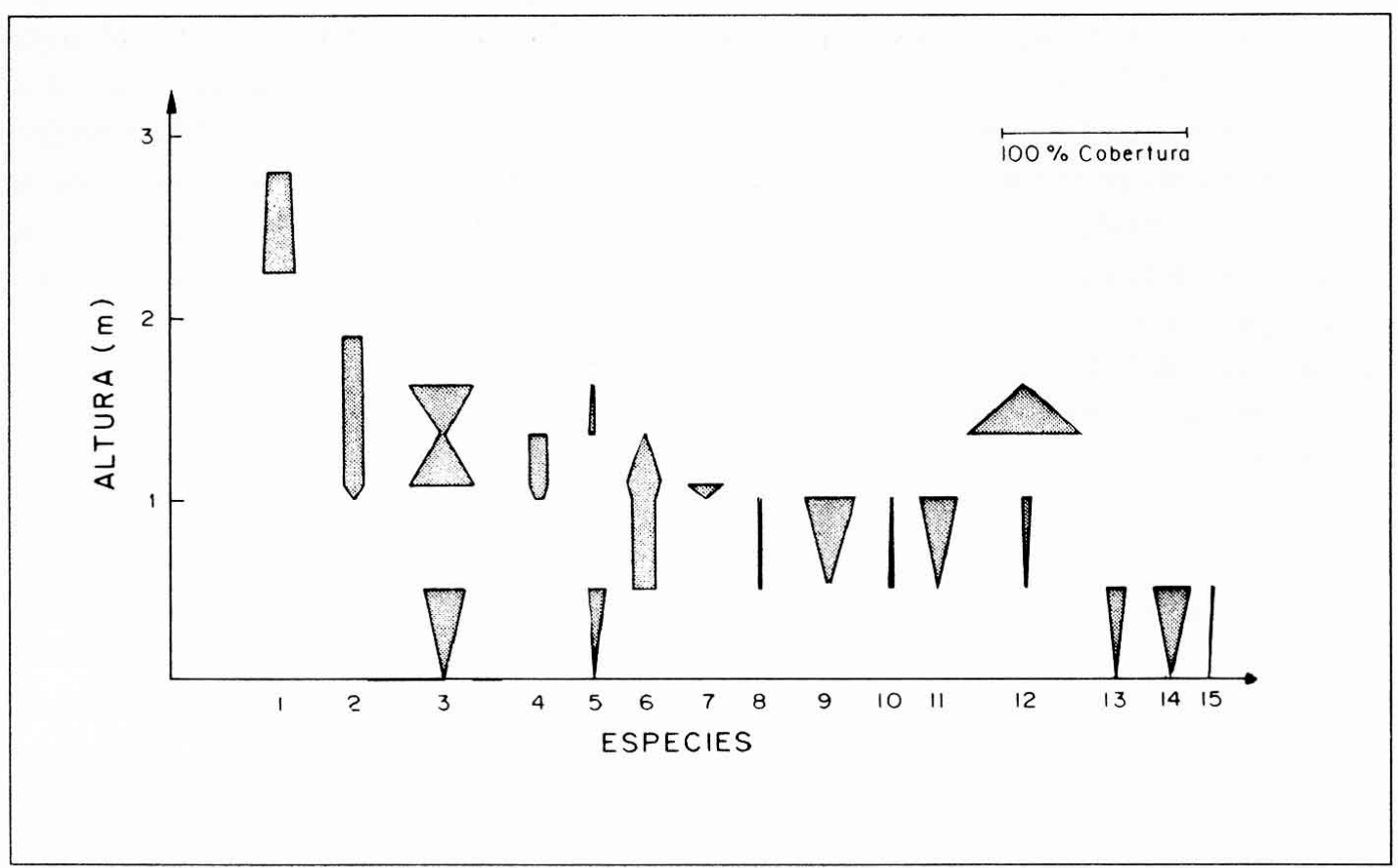

1.- Nodilittorina fernandezensis 2.- Jehlius cirratus 3.- Ulva rigida 4.- Scytothamnus australis 5.- Lithophyllum sp. 6.- Gelidium pseudointrincatum 7.- Heliaster canopus 8.- Polichaeta 9.- Corallina officinalis. va. chilensis 10.- Colpomenia sinuosa 11.- Chondracanthus internedius 12.- Chaetomorpha firma 13.- Dictyota phlyctaenodes 14.- Sphacelaria cirrosa 15.- Lithophylum fernandezianum

Figura 4. Distribución de macroalgas y macroinvertebrados en playa El Palillo, isla Robinson Crusoe.

Figure 4. Distributional patterns of macroalgae and macroinvertebrates in El Palillo beach, Robinson Crusoe island.

\section{DISCUSIÓN}

Los patrones de distribución de los organismos intermareales en estas costas caracterizan un patrón de zonación diferente al conocido para otras áreas geográficas cercanas, como el litoral central de Chile continental. Es notable en la isla la ausencia del cinturón de mitílidos en el intermareal medio y el de las grandes algas pardas que caracteriza la franja límite, intermareal bajo y submareal alto. Ambos cinturones son característicos y están casi siempre presentes en las costas temperadas de ambos hemisferios (Stephenson y Stephenson, 1972).

Es probable que la ausencia de estos cinturones sea consecuencia de varios factores, entre ellos la naturaleza geológica de la roca, estrechez de la zona intermareal debido a la pendiente pronunciada de los roqueríos o simplemente a factores biogeográ- ficos. Este tipo de roca sufre desmembramientos en forma continua por el oleaje en las zonas más expuestas constituyendo así un sustrato inestable no apropiado para el asentamiento y crecimiento de estos organismos sésiles (Skottsberg, 1943).

La zona de litorídinos en el intermareal alto de la isla incluye una sola especie Nodilittorina fernandeziensis, organismo endémico que habita preferentemente las fisuras de las rocas, al igual que los representantes de la familia Littoridinidae en otras latitudes (Reid, 1986).

En resumen, se puede decir que la distribución de organismos marinos en el intermareal rocoso de la isla Robinson Crusoe se caracteriza por la presencia de al menos dos franjas o zonas características, una en el intermareal alto dominada por Nodilittorina fernandezensis y otra zona extensa que caracteriza el intermareal medio y bajo dominada 
por algas que conforman diferentes asociaciones. Los macroinvertebrados se distribuyen preferentemente en pozas a diferentes alturas de la zona intermareal. En el intermareal medio (1 a $2 \mathrm{~m}$ ) la asociación Ulva-Chaetomorpha y Ulva-GelidiumScytothamnus están presentes, en tanto que hacia el intermareal bajo ( 1 a $0,51 \mathrm{~m}$ y más) son notables las asociaciones Gelidium-Corallina-Chondracanthus y Ulva-Sphacelaria. En la zona submareal continúa el cinturón de algas donde predominan algunas algas pardas como Dictyota phlyctaeonodes y Glossophora kunthii, que serían los equivalentes ecológicos del cinturón de laminariales-fucales presentes en las latitudes temperadas de ambos hemisferios. La presencia de estas asociaciones es coincidente, en términos generales, con los antecedentes entregados por Skottsberg (1943) para otras localidades de la isla Robinson Crusoe. Este autor señala por ejemplo para la región alta del intermareal la asociación Enteromorpha-Chondriella-Gelidium o Enteromorpha-Chaetomorpha y en el intermareal bajo la asociación Ulva-algas pardas y GlossophoraLithothamnion o Chaetomorpha-SplachnidiumLithophyllum.

En este estudio es posible precisar de manera más objetiva estas asociaciones, estableciendo las especies dominantes de éstas a diferentes alturas de la zona intermareal. En el caso de la primera asociación este estudio discrepa con Skottsberg (1943) en las especies Chondriella pusilla y Enteromorpha spp como especies dominantes de esta asociación. En este estudio se pudo determinar que $C h$. pusilla crece junto a Gelidium y de la misma forma; sin embargo, es bastante escasa y se confunde con Chondracanthus y otra alga perteneciente a las Phyllophoraceae, tal vez una Ahnfeltiopsis, ambas creciendo junto a Gelidium, pero que en ningún caso se trata de Chondriella. Diferentes especies de Enteromorpha en pozas intermareales altas y Ulva rigida que crece adherida a las rocas a esta altura de mareas. De todas estas especies, la única que se observa a lo largo de toda la isla formando un verdadero cinturón, es la alga verde Chaetomorpha firma en el intermareal medio a bajo, que es bastante resistente al oleaje y está siempre presente en este nivel mareal.

La estrechez de la zona intermareal es limitante para la extensión de los cinturones. Los mitílidos están completamente ausentes y esto puede obedecer a la falta de espacio primario libre más que a razones biogeográficas. La ausencia del cinturón de grandes algas pardas, sorprende aún más, si se con- sidera que en islas de igual origen y edad aproximada como isla de Pascua e islas Desventuradas, este cinturón de algas pardas está presente en esta zona del intermareal. En isla de Pascua, esta franja está representada por Sargassum skottsbergii Sjöested, un representante de las Fucales (Borgesen, 1924) y en islas Desventuradas por Eisenia cokerii Howe (Duhart, com. pers.), alga Laminarial semejante en tamaño a especies de Lessonia y Laminaria.

Por otra parte la biodiversidad de organismos marinos en el intermareal de la isla Robinson Crusoe, en general es alta si se refiere al número total de taxa que habitan esta zona. Como se puede observar, éste es similar o tal vez más alto si se compara con el número de taxa encontrados en roqueríos intermareales de áreas geográficas cercanas del continente (Santelices et al., 1977; Santelices, 1991). Sin embargo, cabe destacar que la representatividad de algunos grupos es comparativamente baja con relación a otras islas de igual superficie o con Chile continental. Por ejemplo, Otaíza y Santelices (1985) citan 22 especies de chitones para Chile central $\left(30^{\circ}\right.$ $40^{\circ} \mathrm{S}$ ), mientras que en el archipiélago de Juan Fernández sólo se han registrado 5 especies. Similar situación ocurre con los Acmaeidae, los que son abundantes y numerosos, más de 11 especies en la costa de Chile Central, y sólo una en el archipiélago que es Acmaea fernandeziana y es endémica.

Con relación a las macroalgas, el número de taxa presentes en la isla es de 123 especies (Ramírez, en prensa). Según Santelices (1992), la flora marina bentónica de Juan Fernández es pobre en especies comparada con otras islas de igual tamaño y edad. Sin embargo, si se compara la riqueza de especies del archipiélago con la costa central de Chile (aprox. 150 especies) o incluso con isla de Pascua (121 especies) que son las localidades geográficas más cercanas, estas cifras no permiten aceptar tal aseveración.

Este trabajo da cuenta por primera vez de la presencia de período reproductivo para dos especies de gastrópodos Nodilittorina fernandezensis y Aplysia parvula juanina, y también por primera vez se registran densidades específicas de $N$. fernandezensis (promedio de 181 indiv. $/ \mathrm{m}^{2}$ ) y del holotúrido Metensiothuria platei en pozas intermareales (promedio de 25 indiv. $/ \mathrm{m}^{2}$ ). Los valores de abundancia de $N$. fernandezensis son inferiores a los registrados para N. pyramidalys pascua en isla de Pascua, 304 indiv. $/ \mathrm{m}^{2}$ (Osorio y Cantuarias, 1989) y también inferiores a los de $N$. peruviana en la costa cen- 
tral de Chile, donde alcanza hasta +100 indiv. $/ 0,25 \mathrm{~m}^{2}$ en octubre, período de máxima abundancia (Santelices et al., 1986). En el caso de M. platei las densidades fueron inferiores a las registradas para Holothuria difficilis y H. cinerascens en isla de Pascua deonde alcanzan promedios de hasta 70 indiv./ $\mathrm{m}^{2}$ (Osorio y Cantuarias, 1989). Las diferencias registradas entre las especies pueden ser atribuidas a períodos estacionales diferentes, características morfológicas de los sustratos o factores biogeográficos. De los 13 equinodermos citados para el archipiélago (Codoceo, 1976), 6 son intermareales. En playa El Palillo, se colectaron 3 de estas 6 especies, todas ellas endémicas del archipiélago.

Un análisis biogeográfico de esta biota permite señalar que los taxa de la flora marina en estas costas incluyen preferentemente elementos de amplia distribución, circunscritos a regiones de aguas temperadas cálidas y elementos endémicos; sumado a ello, también está presente un componente subantártico importante, lo que es coincidente con lo señalado por Santelices (1992). En el caso de la fauna marina, un análisis biogeográfico sobre los crustáceos decápodos (Andrade, 1985), establece que este grupo presenta una estrecha relación con el Pacífico suroriental y a su vez una mayor afinidad con la fauna de crustáceos de las zonas norte y central de Chile, con un endemismo que alcanza al $15 \%$.

Para otros invertebrados marinos, se observa un patrón biogeográfico similar al de Andrade (1985), al cual se sumaría un componente menor de especies con afinidades tropicales. La presencia de estos elementos permite confirmar, por una parte, que el sistema de corrientes que baña la costa de Chile continental alcanza también las costas de este archipiélago, lo que coincide con los resultados obtenidos por Arana (1987) y estaría determinando la composición de especies presentes en estas islas. Por otra parte, también es importante considerar los efectos que haya tenido la incursión de aguas tropicales a través del fenómeno de "El Niño" en la presencia de ciertas especies con afinidades subtropicales o tropicales, como lo señala Santelices (1992). La estrecha relación de la fauna de invertebrados entre Chile continental y el archipiélago postulada por Andrade (1985), puede ser atribuida más al sistema de corrientes que al transporte vía marítima entre la isla y el continente como lo postulara Santelices (1992).

Este trabajo es el primero en aportar datos cuan- titativos sobre las comunidades marinas bentónicas y como tal, los antecedentes aunque preliminares son útiles para futuros estudios sobre estas comunidades intermareales. En este sentido, ellos deben ser considerados como básicos en estudios de monitoreo de este ecosistema marino para detectar cambios naturales o artificiales que afecten la estructura y funcionamiento de estas comunidades marinas.

\section{AGRADECIMIENTOS}

Las autoras agradecen a los especialistas $\mathrm{N}$. Rozbaczylo, O. Gálvez y R. Bieler por su inestimable ayuda en la identificación de las especies de poliquetos, poliplacóforos y vermétidos. Por la colaboración en terreno, agradecemos la ayuda de Juanita Bustamante y Cecilia Alday. Finalmente, agradecemos a CONAF Parque Nacional Juan Fernández por las facilidades otorgadas y en forma especial a los guardaparques Marieta Uribe, Jorge Angulo, Oscar Chamorro, Ramón Schiller, Guillermo Araya, Bernardo López y Manuel Tobar, sin cuyo apoyo logístico hubiese sido imposible desarrollar este trabajo.

\section{REFERENCIAS}

Andrade, H. 1985. Crustáceos decápodos marinos del Archipiélago de Juan Fernández. En: P. Arana (ed.). Investigaciones Marinas en el Archipiélago de Juan Fernández. Escuela de Ciencias del Mar, UCV, Valparaíso, pp. 109-116.

Arana, P. 1987. Perspectivas históricas y proyecciones de la actividad pesquera realizada en el Archipiélago de Juan Fernández, Chile. En: J.C. Castilla (ed.). Islas Oceánicas Chilenas: Conocimiento científico y necesidades de investigaciones. Ediciones Universidad Católica de Chile. Santiago, pp. 319353.

Báez, P. y R. Ruiz. 1985. Crustáceos de las Islas Oceánicas de Chile depositados en el Museo Nacional de Historia Natural Santiago. En: P. Arana (ed.). Investigaciones Marinas en el Archipiélago de Juan Fernández. Escuela de Ciencias del Mar, UCV, Valparaíso, pp. 93-108.

Borgesen, F. 1924. Marine algae from Easter Island. En: C. Skottsberg (ed.). The Natural History of Juan Fernández and Easter Island. Vol. 2, Part 3, 9: 247309. 
Codoceo, M. 1976. Asteroidea, echinoidea and holothuroidea of the Desventuradas and Juan Fernández islands off Chile with new records for the last archipelago. Thalassia Jugoslavica, 12(1): 87-98.

Desqueyroux, R. 1972. Demospongiae (Porifera) de la costa de Chile. Gayana, Zool., 20: 1-71.

Earle, S.A. 1969. Hummbrella, a new red algae of uncertain taxonomic position from the Juan Fernández Islands. Occasional Papers of the Farlow Herbarium of Cryptogamic Botany. Harvard Univ., pp. 1-5.

Etcheverry, H. 1960. Algas marinas de las islas oceánicas chilenas. Rev. Biol. Mar., 10: 83-132.

González-Ferrán, O. 1987. Evolución geológica de las Islas Chilenas en el Océano Pacífico. En: J.C. Castilla (ed.). Islas Oceánicas Chilenas: Conocimiento científico y necesidades de investigaciones. Ediciones Universidad Católica de Chile, Santiago, pp. 37-54.

Levring, T. 1941. Die Meeresalgen der Juan Fernández Islands. En: C. Skottsberg (ed.). The Natural History of Juan Fernández and Eastern Island. Vol. 2, Part 5, 22: 601-670

Müller, D.G. y M.E. Ramírez. 1994. Filamentous brown algae from the Juan Fernández Archipelago (Chile). Contribution of laboratory culture techniques to a phytogeographic survey. Bot. Mar., 37: 205-211.

Odhner, N. 1922. Mollusca from Juan Fernández and Easter Island. En: C. Skottsberg (ed.). The Natural History of Juan Fernández and Easter Island. Vol. 3, Part 2, 22: 219-254.

Osorio, C. y V. Cantuarias. 1989. Vertical distribution of Mollusks on the rocky intertidal of Easter Island. Pacific Sci., 43(4): 302-315.

Otaíza, R.D. y B. Santelices. 1985. Vertical distribution of chitons (Mollusca: Polyplacophora) in the rocky intertidal zone of central Chile. J. Experim. Mar. Biol. Ecol., 86: 229-240.

Ramírez, M.E. 1995. Documentación y puesta en valor de la colección de algas marinas del Archipiélago de Juan Fernández, depositada en el Museo Nacional de Historia Natural. Informes Fondo de Apoyo a la Investigación 1995. Centro de Investigación Barros Arana. DIBAM: 17-22.
Ramírez, M.E. Museos, colecciones y el desafío de la biodiversidad. En: K. Alveal, M.E. Ferrario, E.C. Oliveira y E. Sar (eds.). Biodiversidad Algal. Universidad de Concepción, Chile (en prensa).

Reid, D. 1986. The littorinid molluscs of mangrove forests in the Indo-Pacific region. Brit. Mus. Nat. Hist., 978: 1-227.

Rozbaczylo, N. y J.C. Castilla. 1987. Invertebrados marinos del Archipiélago de Juan Fernández. En: J.C. Castilla (ed.). Islas Oceánicas Chilenas: Conocimiento científico y necesidades de investigaciones. Ediciones Universidad Católica de Chile, Santiago, pp. 167-189.

Santelices, B. 1987. Flora marina bentónica de las Islas Oceánicas Chilenas. En: J.C. Castilla (ed.). Islas Oceánicas Chilenas: Conocimiento científico y necesidades de investigaciones. Ediciones Universidad Católica de Chile, Santiago, pp. 101-126.

Santelices, B. 1991. Intertidal and littoral ecocystems. En: A.C. Mathieson y P.H. Nienhuis (eds.). Ecosystems of the World. Elsevier, Amsterdam, pp. 347-369.

Santelices, B. 1992. Marine phytogeography of the Juan Fernández Archipelago: A new assessment. Pacific Sci., 46(4): 438-452.

Santelices, B., J. Vásquez y I. Meneses. 1986. Patrones de distribución y dietas de un gremio de moluscos herbívoros en hábitats intermareales expuestos de Chile Central. En: B. Santelices (ed.). Ediciones Universidad Católica de Chile. Santiago. Monografías Biológicas, 4: 147-171.

Santelices, B., J. Cancino, S. Montalva, R. Pinto y E. González. 1977. Estudios ecológicos en la zona costera afectada por contaminación del "Northern Breeze". II. Comunidades de playas de rocas. Medio Ambiente, 2(2): 65-83.

Skottsberg, C. 1943. Marine algal communities of the Juan Fernández Island, with remarks on the composition of the flora. En: C. Skottsberg (ed.). The Natural History of Juan Fernández and Easter Island. Vol. 2, Part 5, 23: 671-696.

Stephenson, T.A. y A. Stephenson. 1972. Life between tidemarks on rocky shores. Freeman, San Francisco, 425 pp. 